Supporting Information

\title{
Development of a fast chemiluminescent magneto-immunoassay for sensitive Plasmodium falciparum detection in whole blood
}

\author{
Ana Sánchez-Cano ${ }^{1,2}$, Gisela Ruiz-Vega ${ }^{{ }^{*}}$, Sergi Vicente-Gómez ${ }^{1}$, Erica de la Serna ${ }^{1}$, Elena \\ Sulleiro ${ }^{2,3,4}$, Israel Molina ${ }^{4,5}$, Adrián Sánchez-Montalvá ${ }^{2,4,5}$, Eva Baldrich $^{1^{*}}$. \\ ${ }^{1}$ Diagnostic Nanotools Group, Vall d'Hebron Institut de Recerca (VHIR), Vall d'Hebron Barcelona \\ Hospital Campus, Barcelona, 08035, Spain. \\ ${ }^{2}$ Universitat Autònoma de Barcelona (UAB), Bellaterra, 08193, Spain \\ ${ }^{3}$ Microbiology Department, Vall d'Hebron Hospital Universitari, Vall d'Hebron Barcelona Hospital \\ Campus, Barcelona, 08035, Spain. \\ ${ }^{4}$ PROSICS (Catalan International Health Program), Barcelona, 08035, Spain \\ ${ }^{5}$ Infectious Diseases Department, Vall d'Hebron Hospital Universitari, Vall d'Hebron Barcelona Hospital \\ Campus, Barcelona, 08035, Spain. \\ *corresponding author: eva.baldrich@vhir.org; gisela.ruiz@vhir.org
}

\section{Table of Contents}

\section{Material and Methods}

Production of biotinylated detection antibodies (bd-MAb).

MB immuno-modification

\section{Tables}

Table S1. Examples of magneto-immunoassays reported before for malaria diagnosis.

Table S2. Immunoassays and immunosensors reported using Poly-HRP for signal enhancement.

Table S3. Summary of the protocols employed in this work for conjugation of MB with cMAb using EDC.

\section{Figures}

Figure S1. Scheme and protocol of the classical sandwich ELISA used for PfLDH detection.

Figure S2. Single PfLDH immunocapture optimization time.

Figure S3. Performance of the 2-step magneto-immunoassay when using alternatively two different types of commercial MB, Dynabeads and Sera-Mag.

Figure S4. Performance of Sera-Mag and Dynabeads MB submitted to different blocking protocols during the immuno-modification (Protocol 2).

Figure S5. Performance of the 2-step magneto-immunoassay in lysed whole blood. 


\section{Material and Methods}

Production of biotinylated detection antibodies (bd-MAb).

The detection antibody (d-MAb) was first subjected to a buffer exchange in order to remove interfering reagents, such as sodium azide. For this, $300 \mu \mathrm{g}$ of d-MAb were placed in an Amicon ${ }^{\circledR}$ Ultra $0.5 \mathrm{~mL}$ centrifuge filter (Merck Millipore) in a final volume of $0.5 \mathrm{~mL}$. The device was centrifuged at $14,000 \times \mathrm{g}$ for $10 \mathrm{~min}$, filled with $0.5 \mathrm{~mL}$ of carbonate-bicarbonate buffer $(0.1 \mathrm{M}$, $\mathrm{pH}$ 9.5), and centrifuged again. This procedure was repeated once more. The concentrated dMAb was then recovered and carbonate-bicarbonate buffer $(0.1 \mathrm{M}, \mathrm{pH} 9.5)$ was added to bring the antibody to the initial concentration.

The d-MAb was then biotinylated using 6-((6-((biotinoyl)amino)hexanoyl)amino)hexanoic acid, sulfosuccinimidyl ester, sodium salt (biotin-XX, SSE; Ref. B6352, Thermo Fisher Scientific). For this, a biotin-XX SSE stock was prepared at a concentration of $2.5 \mathrm{mg} \mathrm{mL}^{-1}$ in Milli-Q water and $9.66 \mu \mathrm{L}$ were added to the d-MAb. The mixture was stirred at $24^{\circ} \mathrm{C}$ in the dark for $2 \mathrm{~h}$. The biotin not bound to the d-MAb was eliminated using a PD G25 exclusion column (GE Healthcare) following the provider's instructions. The bd-MAb was finally diluted to a concentration of 150 $\mu \mathrm{g} \mathrm{mL} \mathrm{L}^{-1}$ with $1 \% \mathrm{BSA}$ and was stored at $-20^{\circ} \mathrm{C}$ in working aliquots until it was used.

\section{MB immuno-modification}

$M B$ were modified with $c-M A b$ using the protocols recommended by the corresponding suppliers, as well as a customized protocol (Table S-3). In all cases, incubations were performed in agitation using either a vortex (Vortex Genie 2, Scientific Industries, Bohemia, USA) or a thermoshaker (Thermal Shake lite, VWR, Barcelona, Spain).

Protocol 1 (Dynabeads). MB (20 $\mu \mathrm{g}$ in $200 \mu \mathrm{L}$ ) were washed twice with $15 \mathrm{mM}$ MES using a magnetic separator (BILATEST, Sigma Aldrich). MB were next vortexed for $120 \mathrm{~min}$ in $100 \mu \mathrm{L}$ of MES containing $50 \mu \mathrm{g}$ of $\mathrm{c}-\mathrm{MAb}$ and $4 \mathrm{mg} \mathrm{mL}^{-1}$ of EDC (600 rpm). After that, MB were serially washed with $200 \mu \mathrm{L}$ of MES and with $200 \mu \mathrm{L}$ of PBS, and were blocked for $1 \mathrm{~h}$ with PBS, $1 \%$ BSA (PBS-BSA1\%). The c-MAb-MB were then washed twice for 5 min with $100 \mu \mathrm{L}$ of PBS, $0.1 \%$ Tween 20 (PBST0.1\%) and resuspended in $1 \mathrm{~mL}$ of PBST0.1\%, 0.2\% BSA (PBST0.1\%-BSA0.2\%) for storage at $4{ }^{\circ} \mathrm{C}$ (final concentration of $1.4-2.4 \times 10^{9} \mathrm{MB} \mathrm{mL}^{-1}$, equivalent to $2 \mathrm{mg} \mathrm{mL}^{-1}$ ). 
Protocol 2 (Dynabeads-based customized protocol). The protocol was similar to Protocol 1, but conjugation was executed for $15 \mathrm{~min}$ at $950 \mathrm{rpm}$ in a thermoshaker.

Protocol 3 (Sera-Mag). MB (25 $\mu \mathrm{g}$ in $50 \mu \mathrm{L})$ were washed twice with $100 \mathrm{mM}$ MES using a magnetic separator. MB were next vortexed for 15 min with $25 \mu \mathrm{g}$ of c-MAb in $470 \mu \mathrm{L}$ of MES (600 rpm), $30 \mu \mathrm{L}$ of $10 \mathrm{mg} \mathrm{mL}^{-1}$ EDC were added, and the mixture was vortexed for $1 \mathrm{~h}$ more. Then, MB were serially washed with $200 \mu \mathrm{L}$ of MES and with $200 \mu \mathrm{L}$ of PBS, and were blocked for $1 \mathrm{~h}$ with PBS-BSA1\%. The c-MAb-MB were then washed twice for $5 \mathrm{~min}$ with $100 \mu \mathrm{L}$ of PBST0.1\% and were resuspended in $1 \mathrm{~mL}$ of PBST-BSA0.2\% for storage at $4{ }^{\circ} \mathrm{C}$ (final concentration of $4.77 \times 10^{9} \mathrm{MB} \mathrm{mL}^{-1}$, equivalent to $5 \mathrm{mg} \mathrm{mL}^{-1}$ ).

Protocol 4 (ProMag). MB (100 $\mu \mathrm{L} ; 25.2 \mu \mathrm{g}$ for ProMag Series 3 and $24 \mu \mathrm{g}$ for ProMag Series 1) were washed twice with $100 \mathrm{mM}$ MES using a magnetic separator. MB were next agitated in a thermoshaker for 15 min with $10 \mathrm{mg} \mathrm{mL}^{-1} \mathrm{EDC}$ (950 rpm). After that, MB were washed twice with $500 \mu \mathrm{L}$ of PBS, were resuspended in $1 \mathrm{~mL}$ of PBS with $56.3 \mu \mathrm{g}$ of c-MAb, and were incubated for $3 \mathrm{~h}$ (thermoshaker, $950 \mathrm{rpm}$ ). The MB were next transferred to $1 \mathrm{~mL}$ of $40 \mathrm{mM}$ ethanolamine in MiliQ water supplemented with 1\% BSA and were vortexed for $30 \mathrm{~min}(600 \mathrm{rpm})$. The c-MAbMB were then washed with $1 \mathrm{~mL}$ of PBS-BSA1\% and were resuspended in $1260 \mu \mathrm{L}$ of PBS, $0.1 \%$ BSA (PBS-BSA0.1\%) for storage at $4{ }^{\circ} \mathrm{C}$ (final concentration of $2 \mathrm{mg} \mathrm{mL}^{-1}$ ). 
Table S1. Examples of magneto-immunoassays reported before for malaria diagnosis. The number of assay steps and assay time include the different incubations, but not the washes, which are not detailed in all the works reviewed, or detection, which is stated separately. QD, quantum dots; PLDH, Plasmodium lactate dehydrogenase (LDH); PfLDH, Plasmodium falciparum LDH; HRPII, histidine rich protein.

* According to the provider, the recombinant PfLDH protein used in this work has a molecular weight of approximately $65 \mathrm{kDa}$.

\begin{tabular}{|c|c|c|c|c|c|c|c|c|c|c|}
\hline $\begin{array}{c}\text { Assay } \\
\text { format }\end{array}$ & Detection method & Target & $\begin{array}{l}\text { MB modif. } \\
\text { time }\end{array}$ & $\begin{array}{l}\text { Assay } \\
\text { steps }\end{array}$ & $\begin{array}{l}\text { Assay } \\
\text { time }\end{array}$ & $\begin{array}{l}\text { Detection } \\
\text { time }\end{array}$ & Linear assay range & LOD & Samples & Ref. \\
\hline Direct & Colorimetric & PfLDH & $1 \mathrm{~h}$ & 2 & $45 \mathrm{~min}$ & $30 \mathrm{~min}$ & - & $25.7 \pm 1.1 \mathrm{pM}$ & $\begin{array}{l}\text { Parasitized } \\
\text { whole blood }\end{array}$ & 1 \\
\hline Sandwich & Colorimetric & $\begin{array}{l}\text { PLDH } \\
\text { PfHRP-II }\end{array}$ & $1 \mathrm{~h}$ & 2 & $\begin{array}{l}35 \mathrm{~min} \\
25 \mathrm{~min}\end{array}$ & $15 \mathrm{~min}$ & $\begin{array}{l}7.0-500 \mathrm{pM} \\
1.5-80 \mathrm{pM}\end{array}$ & $\begin{array}{l}2.6 \pm 1.5 \mathrm{pM} \\
1.6 \pm 1.0 \mathrm{pM}\end{array}$ & $\begin{array}{l}\text { Commercial } \\
\text { pooled human } \\
\text { whole blood }\end{array}$ & 2 \\
\hline Sandwich & Colorimetric & HRP-II & $36 \mathrm{~h}$ & 1 & $2 \mathrm{~h}$ & $30 \mathrm{~min}$ & $1.31-62.5 \mathrm{ng} \mathrm{mL}^{-1}$ & $1.31 \mathrm{ng} \mathrm{mL}^{-1}$ & $\begin{array}{l}12 \text { patient } \\
\text { serum } \\
\text { samples }\end{array}$ & 3 \\
\hline Sandwich & Fluorescent (QD) & $\begin{array}{l}\text { PfLDH / } \\
\text { PvLDH }\end{array}$ & $3 \mathrm{~h}$ & 3 & $\begin{array}{l}110 \\
\min \end{array}$ & $5 \mathrm{~min}$ & $0.51-15 \mathrm{fmols}$ & $\begin{array}{c}0.001-0.066 \mathrm{ng} \mathrm{mL}^{-1} \\
(10-1000 \mathrm{amols})\end{array}$ & - & 4 \\
\hline Sandwich & $\begin{array}{c}\text { Colorimetric } \\
\text { Fluorescent } \\
\text { Chemiluminescent }\end{array}$ & PfLDH & $1 \mathrm{~h} 25 \mathrm{~min}$ & 2 & $10 \mathrm{~min}$ & $\begin{array}{l}20 \mathrm{~min} \\
15 \mathrm{~min} \\
1 \mathrm{~min}\end{array}$ & $\begin{array}{c}0.4-12.5 \mathrm{ng} \mathrm{mL}^{-1} \\
0.1-25 \mathrm{ng} \mathrm{mL}^{-1} \\
0.04-12.5 \mathrm{ng} \mathrm{mL}^{-1}\end{array}$ & $\begin{array}{l}0.12 \mathrm{ng} \mathrm{mL}^{-1}(1.85 \mathrm{pM})^{*} \\
0.11 \mathrm{ng} \mathrm{mL}^{-1}(1.69 \mathrm{pM})^{*} \\
0.02 \mathrm{ng} \mathrm{mL}^{-1}(0.31 \mathrm{pM})^{*}\end{array}$ & $\begin{array}{l}7 \text { patient } \\
\text { whole blood } \\
\text { samples }\end{array}$ & $\begin{array}{l}\text { This } \\
\text { work }\end{array}$ \\
\hline
\end{tabular}


Table S2. Immunoassays and immunosensors reported using Poly-HRP for signal enhancement. Assay steps and assay time include incubations but not washes, which are not detailed in all the works reviewed, or detection, which is stated separately. Ab, antibody; DENV (1-4), dengue virus (serotype 1-4); DPV, differential pulse voltammetry; DWCNT, double-walled CNT; HBV, Hepatitis B virus; HER-2, human epidermal growth factor receptor 2; IL-1 $\beta$, interleukin-1beta ; MMP-9, matrix metallopeptidase 9; NS1, nonstructural protein 1; sLFIA, lateral flow immunoassay; ON, overnight; PfLDH, Plasmodium falciparum lactate dehydrogenase ; pPPA, poly(pyrrole propionic acid); SPCE, screen-printed carbon electrode; SWCNT, single-walled CNT; TGF- $\beta 1$; transforming growth factor beta 1 ; TNF- $\alpha$, tumour necrosis factor.

\begin{tabular}{|c|c|c|c|c|c|c|c|c|c|}
\hline Assay format & Detection type & Analyte & $\begin{array}{l}\text { Time of } \\
\text { functionalization }\end{array}$ & $\begin{array}{l}\text { Assay } \\
\text { steps }\end{array}$ & $\begin{array}{l}\text { Assay } \\
\text { time }\end{array}$ & $\begin{array}{l}\text { Detection } \\
\text { time }\end{array}$ & LOD & Samples & Ref. \\
\hline $\begin{array}{l}\text { Electrochemical } \\
\text { immunosensor }\end{array}$ & $\begin{array}{l}\text { Amperometric (SPCE } \\
\text { with MWCNT-Ab1) }\end{array}$ & TGF- $\beta 1$ & $\begin{array}{l}\mathrm{ON}+1 \mathrm{~h} 30 \mathrm{~min} \\
\text { (SPCE) }\end{array}$ & 3 & $\begin{array}{l}1 \mathrm{~h} 20 \\
\min \end{array}$ & $<5 \min$ & $0.0013 \mathrm{ng} \mathrm{mL}^{-1}$ & 2 serum samples & 6 \\
\hline $\begin{array}{l}\text { Electrochemical } \\
\text { immunosensor }\end{array}$ & $\begin{array}{l}\text { Amperometric (SPCE } \\
\text { with Phe-DWCNT) }\end{array}$ & $\begin{array}{l}\text { IL-1 } \beta \text { and } \\
\text { TNF- } \alpha\end{array}$ & $\begin{array}{l}2 \text { h } 30 \text { min (Phe- } \\
\text { DWCNT-SPE) }\end{array}$ & 3 & $2 \mathrm{~h}$ & $<5 \min$ & $\begin{array}{l}\left.0.00038 \mathrm{ng} \mathrm{mL}^{-1} \text { (IL-1 } \beta\right) \\
0.00085 \mathrm{ng} \mathrm{mL}^{-1}(\mathrm{TNF}-\alpha)\end{array}$ & $\begin{array}{l}\text { Spiked serum } \\
\text { samples and } 2 \\
\text { saliva samples }\end{array}$ & 7 \\
\hline $\begin{array}{l}\text { Electrochemical } \\
\text { immunosensor }\end{array}$ & $\begin{array}{l}\text { Amperometric } \\
\text { (inkjet-printed gold } \\
8 x \text { array) }\end{array}$ & HER-2 & $\begin{array}{l}24 h+O N(A b)+ \\
1 h\end{array}$ & 3 & $15 \mathrm{~min}$ & $3 \mathrm{~min}$ & $0.012 \mathrm{ng} \mathrm{mL}^{-1}$ & $\begin{array}{l}\text { Spiked serum } \\
\text { samples }\end{array}$ & 8 \\
\hline $\begin{array}{l}\text { Electrochemical } \\
\text { immunosensor }\end{array}$ & $\begin{array}{l}\text { Amperometric (pPPA } \\
\text { electrode) }\end{array}$ & $\begin{array}{l}\text { Endoglin } \\
\text { (CD105) }\end{array}$ & $2 \mathrm{~h}$ & 3 & $\begin{array}{l}1 \mathrm{~h} 45 \\
\min \end{array}$ & $3 \min$ & $0.14 \mathrm{ng} \mathrm{mL}^{-1}$ & $\begin{array}{l}16 \text { serum } \\
\text { samples }\end{array}$ & 9 \\
\hline $\begin{array}{l}\text { Electrochemical } \\
\text { immunosensor }\end{array}$ & $\begin{array}{l}\text { Amperometric } \\
\text { (SPCE) }\end{array}$ & MMP-9 & $\mathrm{ON}+45 \mathrm{~min}$ & 2 & $40 \mathrm{~min}$ & $5 \mathrm{~min}$ & $0.018-0.028 \mathrm{pg} \mathrm{mL}^{-1}$ & $\begin{array}{l}15 \text { plasma } \\
\text { samples \& } 6 \\
\text { uterine aspirates }\end{array}$ & 10 \\
\hline $\begin{array}{l}\text { Electrochemical } \\
\text { magneto- } \\
\text { immunosensor }\end{array}$ & $\begin{array}{l}\text { Amperometric } \\
\text { (SPCE) }\end{array}$ & TGF- $\beta 1$ & $3 \mathrm{~h} 4 \mathrm{~min}(\mathrm{MB})$ & 3 & $\begin{array}{l}2 \mathrm{~h} 20 \\
\min \end{array}$ & $<5 \min$ & $0.010 \mathrm{ng} \mathrm{mL}^{-1}$ & $\begin{array}{l}3 \text { spiked urine } \\
\text { samples }\end{array}$ & 11 \\
\hline
\end{tabular}




\begin{tabular}{|c|c|c|c|c|c|c|c|c|c|}
\hline $\begin{array}{l}\text { Electrochemical } \\
\text { magneto- } \\
\text { immunosensor }\end{array}$ & $\begin{array}{l}\text { Amperometric } \\
\text { (SPCE) }\end{array}$ & MMP-9 & $1 \mathrm{~h} 40 \mathrm{~min}(\mathrm{MB})$ & 1 & $30 \mathrm{~min}$ & ns & $0.0024 \mathrm{ng} \mathrm{mL}^{-1}$ & $\begin{array}{l}15 \text { patient serum } \\
\text { samples }\end{array}$ & 12 \\
\hline $\begin{array}{l}\text { Electrochemical } \\
\text { magneto- } \\
\text { immunosensor }\end{array}$ & $\begin{array}{l}\text { Amperometric } \\
\text { (SPCE) }\end{array}$ & MMP-9 & $3 \mathrm{~h}(\mathrm{MB})$ & 1 & $5 \min$ & $5 \min$ & $0,013 \mathrm{ng} \mathrm{mL}^{-1}$ & $\begin{array}{l}10 \text { patient plasma } \\
\text { samples }\end{array}$ & 13 \\
\hline $\begin{array}{l}\text { Electrochemical } \\
\text { magneto- } \\
\text { immunosensor }\end{array}$ & $\begin{array}{l}\text { Amperometric } \\
\text { (paper microfluidic } \\
\text { SPCE) }\end{array}$ & PfLDH & $3 \mathrm{~h}(\mathrm{MB})$ & 1 & $5 \mathrm{~min}$ & $5 \mathrm{~min}$ & $2.51 \mathrm{ng} \mathrm{mL}^{-1}$ & $\begin{array}{l}3 \text { patient blood } \\
\text { samples }\end{array}$ & 5 \\
\hline $\begin{array}{l}\text { Magneto- } \\
\text { enzyme LFIA }\end{array}$ & $\begin{array}{l}\text { Naked eye } \\
\text { interpretation }\end{array}$ & DENV NS1 & $\begin{array}{l}4 \mathrm{~h} 30 \text { min (MB } \\
\text { modification) / } 2 \\
\mathrm{~h} \text { (test strips) }\end{array}$ & 3 & $25 \mathrm{~min}$ & $10 \mathrm{~min}$ & $\begin{array}{l}0.25 \mathrm{ng} \mathrm{mL}^{-1} \text { (DENV-1 \& - } \\
\text { 3) } \\
0.1 \mathrm{ng} \mathrm{mL}^{-1} \text { (DENV-2) } \\
1 \mathrm{ng} \mathrm{mL}^{-1} \text { (DENV-4) }\end{array}$ & $\begin{array}{l}120 \text { serum } \\
\text { samples }\end{array}$ & 14 \\
\hline ELISA & Colorimetric & MMP-9 & $\mathrm{ON}+1 \mathrm{~h}$ & 2 & $40 \mathrm{~min}$ & $20 \mathrm{~min}$ & $0.001-0.012 \mathrm{ng} \mathrm{mL}^{-1}$ & $\begin{array}{l}38 \text { plasma } \\
\text { samples \& } 16 \\
\text { uterine aspirates }\end{array}$ & 10 \\
\hline ELISA & Colorimetric & PfLDH & $2 \mathrm{~h}$ & 2 & $40 \mathrm{~min}$ & $20 \mathrm{~min}$ & $0.11 \mathrm{ng} \mathrm{mL}^{-1}$ & $\begin{array}{l}11 \text { patient blood } \\
\text { samples }\end{array}$ & 15 \\
\hline $\begin{array}{l}\text { Magneto- } \\
\text { immunoassay }\end{array}$ & Colorimetric & MMP-9 & $2 \mathrm{~h} 30 \min (\mathrm{MB})$ & 2 & 10 & $20 \mathrm{~min}$ & $0.011-0.030 \mathrm{ng} \mathrm{mL}^{-1}$ & $\begin{array}{l}10 \text { patient plasma } \\
\text { samples }\end{array}$ & 16 \\
\hline $\begin{array}{l}\text { Magneto- } \\
\text { immunoassay }\end{array}$ & Chemiluminescent & PfLDH & $1 \mathrm{~h} 25 \mathrm{~min}$ & 2 & $10 \mathrm{~min}$ & $1 \mathrm{~min}$ & $0.02 \mathrm{ng} \mathrm{mL}^{-1}$ & $\begin{array}{l}7 \text { patient blood } \\
\text { samples }\end{array}$ & This work \\
\hline
\end{tabular}


Table S3. Summary of the protocols employed in this work for conjugation of MB with c-MAb using EDC.

\begin{tabular}{|c|c|c|c|c|c|c|}
\hline \multirow[b]{2}{*}{ Protocol } & \multicolumn{3}{|c|}{ Immunoconjugation } & \multicolumn{2}{|c|}{ Blocking } & \multirow{2}{*}{$\begin{array}{l}\text { Washing } \\
\text { Buffer }\end{array}$} \\
\hline & $\mu \mathrm{g} \mathrm{MB}$ & $\mu \mathrm{g} \mathrm{Ab} / \mathrm{mg} \mathrm{MB}$ & $\begin{array}{c}\text { Time } \\
\text { (agitation) }\end{array}$ & Buffer & Time & \\
\hline $\begin{array}{l}\text { Protocol } 1 \text { (Dynabeads } \\
\text { provider) }\end{array}$ & $20 \mu \mathrm{g}$ in $200 \mu \mathrm{L}$ & 25 & $\begin{array}{l}120 \mathrm{~min} \\
\text { (vortex) }\end{array}$ & PBS-BSA1\% & $1 \mathrm{~h}$ & PBST0.1\% \\
\hline $\begin{array}{l}\text { Protocol } 2 \text { (Dynabeads } \\
\text { customized) }\end{array}$ & $20 \mu \mathrm{g}$ in $200 \mu \mathrm{L}$ & 25 & $\begin{array}{c}15 \text { min } \\
\text { (thermoshaker) }\end{array}$ & PBS-BSA1\% & $1 \mathrm{~h}$ & PBST0.1\% \\
\hline $\begin{array}{l}\text { Protocol } 3 \text { (Sera-Mag } \\
\text { provider) }\end{array}$ & $25 \mu \mathrm{g}$ in $50 \mu \mathrm{L}$ & 61.76 & $\begin{array}{c}15 \min +60 \min \\
\text { (vortex) }\end{array}$ & PBS-BSA1\% & $1 \mathrm{~h}$ & PBST0.1\% \\
\hline $\begin{array}{l}\text { Protocol } 4 \text { (ProMag } \\
\text { provider) }\end{array}$ & $\begin{array}{c}25.2 \mu \mathrm{g} \text { in } 100 \mu \mathrm{L} \\
\text { (Series } 3 \text { ) } \\
24 \mu \mathrm{g} \text { in } 100 \mu \mathrm{L} \\
\text { (Series } 1 \text { ) }\end{array}$ & $\begin{array}{l}22.34 \text { (Series 3) } \\
23.46(\text { Series 1) }\end{array}$ & $\begin{array}{c}15 \min +180 \\
\min \\
\text { (vortex) }\end{array}$ & $\begin{array}{c}40 \mathrm{mM} \text { ethanolamine, } \\
1 \% \mathrm{BSA}\end{array}$ & $30 \mathrm{~min}$ & PBS-BSA1\% \\
\hline
\end{tabular}


Figure S1. Scheme and protocol of the classical sandwich ELISA used for PfLDH detection.

Unless otherwise stated, all the incubations were performed with $100 \mu \mathrm{L}$ of PBS-BSA1\% solution per well, for $1 \mathrm{~h}$ each, inside an incubator thermostatized at $35^{\circ} \mathrm{C}$, and were followed by three washes with PBST0.05\% (with $200 \mu \mathrm{L}$ per well each). Briefly, microtiter plates were modified with c-MAb (2.5 $\mu \mathrm{g} \mathrm{mL}^{-1}$ in PBS) and were blocked with PBS-BSA1\%. After washing, the plates were incubated with $0.0016-100 \mathrm{ng} \mathrm{mL}^{-1}$ of PfLDH. Plates were next incubated with $100 \mathrm{ng} \mathrm{mL}^{-1}$ of bd-MAb. Finally, the plates were incubated at room temperature with strep-HRP (diluted 1:200 in PBS-BSA1\%) for $20 \mathrm{~min}$. After four more washes, the plates were incubated for $20 \mathrm{~min}$ at room temperature with TMB (100 $\mu \mathrm{L}$ per well), the reaction was stopped with acid ( $50 \mu \mathrm{L}$ per well), and colour was detected at $450 \mathrm{~nm}$ using an ELISA plate reader.

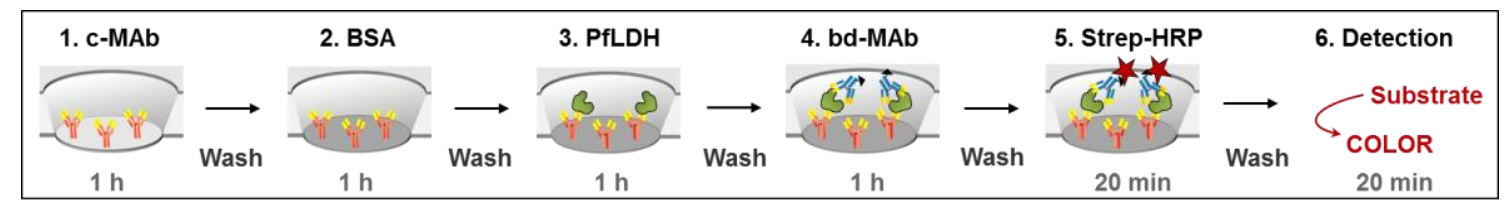


Figure S2. Single PfLDH immunocapture optimization time. The 2-step magneto-immunoassay included a combined incubation of the sample with c-MAb-MB and bd-MAb, which substituted the two consecutive incubations carried in the 3-step magneto-immunoassay. Incubation times of $30 \mathrm{~min}, 15 \mathrm{~min}$ and $5 \mathrm{~min}$ were studied. Figure S-2a summarizes the signals registered for the PfLDH concentrations studied $\left(0.8-100 \mathrm{ng} \mathrm{mL}^{-1}\right)$, comparing the performance of the 2-step and 3-step magneto-immunoassays, in both cases detected using Strep-HRP. The signals registered for the single immunocapture decreased proportionally to the incubation time, as did assay sensitivity in terms of slope. In contrast, the linear range was wider for shorter immunocaptures (Figure S-2a, Insert). The best LOD and LOQ were obtained for the 15-min immunocapture (0.12 and $0.55 \mathrm{ng} \mathrm{mL}^{-1}$, respectively). However, although the 5-min immunocapture produced signals $50 \%$ lower in average for all the concentrations of PfLDH tested, as well as slightly worse LOD/LOQ (Figure S-2b), it still detected the whole range of concentrations of PfLDH studied here, displayed also the widest linear range and was shorter (45 min plus washes), which made it a good candidate for the development of a rapid diagnostic tool.

a

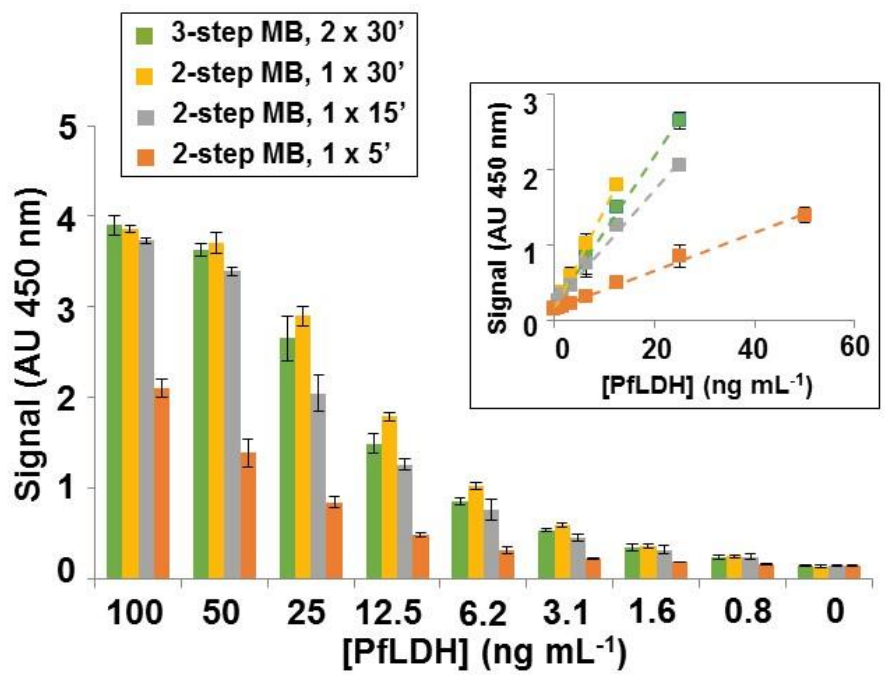

b

\begin{tabular}{|c|c|c|c|c|}
\hline & 3-step, 2 × 30' & 2-step, 1 x 30' & 2-step, 1 x 15' & 2-step, 1 x 5', \\
\hline LOD (ng mL $\left.\mathrm{m}^{-1}\right)$ & 0.23 & 0.30 & 0.12 & 0.49 \\
\hline LOQ (ng mL-1) & 1.00 & 0.95 & 0.85 & 1.39 \\
\hline Linear range $\left(\mathrm{ng} \mathrm{mL}^{-1}\right)$ & $0.78-25$ & $0.78-12.5$ & $0.78-25$ & $0.78-50$ \\
\hline $\begin{array}{c}\text { Linear Equation } \\
\mathbf{R}^{\mathbf{2}}\end{array}$ & $\begin{array}{c}0.099 x+0.197 \\
0.998\end{array}$ & $\begin{array}{c}0.132 x+0.160 \\
0.998\end{array}$ & $\begin{array}{c}0.076 x+0.219 \\
0.991\end{array}$ & $\begin{array}{c}0.025 x+0.160 \\
0.997\end{array}$ \\
\hline Assay time (min) & 100 & 70 & 55 & 45 \\
\hline
\end{tabular}


Figure S3. Performance of the 2-step magneto-immunoassay when using alternatively two different types of commercial MB, Dynabeads and Sera-Mag. Signals (a), S/N ratios (inset) and figures of merit (b) of the 2-step magneto-immunoassay carried using alternatively 3 types of cMAb-MB. Dynabeads were modified with c-MAb using the customized protocol (Protocol 2 in the Materials and Method section of the main manuscript). In the case of Sera-Mag, c-MAb were conjugated alternatively with the customized Dynabeads-based protocol (Protocol 2) and the protocol recommended by the corresponding supplier (Protocol 3).

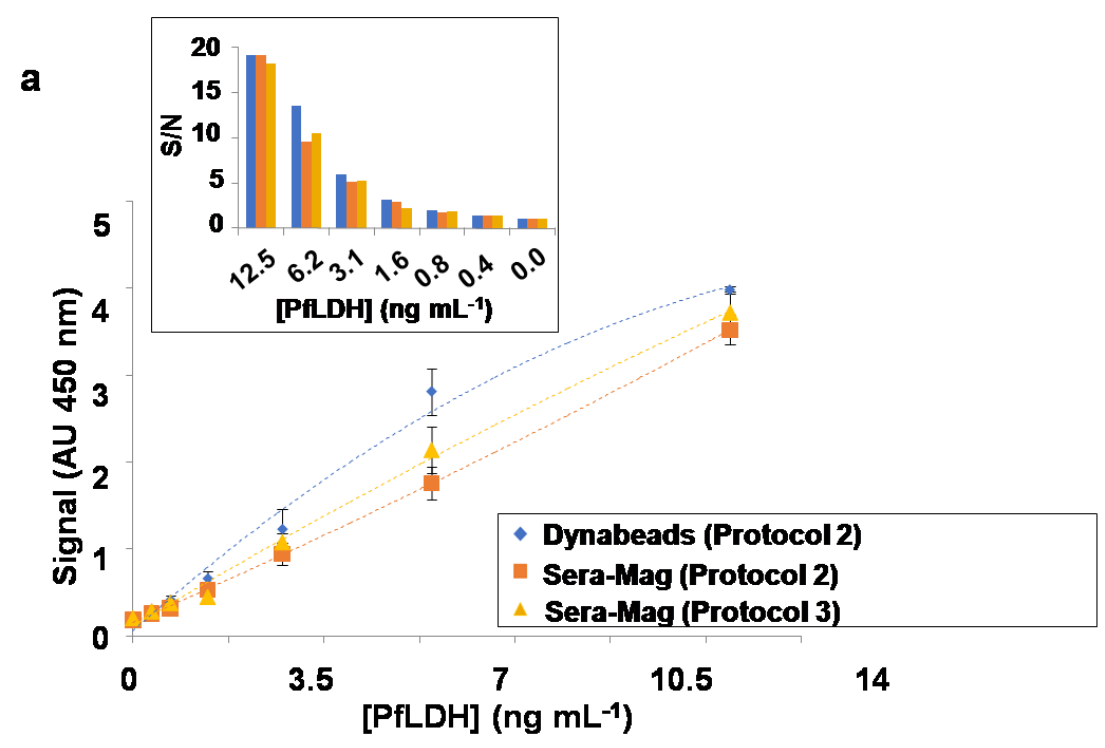

b

\begin{tabular}{|c|c|c|c|}
\hline (ng mL-1) & $\begin{array}{c}\text { Dynabeads } \\
\text { (Protocol 2) }\end{array}$ & $\begin{array}{c}\text { Sera-Mag } \\
\text { (Protocol 2) }\end{array}$ & $\begin{array}{c}\text { Sera-Mag } \\
\text { (Protocol 3) }\end{array}$ \\
\hline LOD & 0.41 & 0.28 & 0.36 \\
\hline LOQ & 0.68 & 0.71 & 0.58 \\
\hline
\end{tabular}


Figure S4. Performance of Sera-Mag and Dynabeads MB submitted to different blocking protocols during the immuno-modification (Protocol 2).

Sera-Mag MB blocking was re-optimized in an attempt to reduce the background noise. Results displayed in Figure S-3a show that extending the blocking with BSA $1 \%$ from $1 \mathrm{~h}$ to overnight (ON) contributed to reduce $20 \%$ the background noise and the LOQ. In contrast, augmenting BSA concentration during ON blocking seemed to revert this effect, presumably due to an excess of protein sheltering the $\mathrm{c}-\mathrm{MAb}$ and interfering in immunobinding. Other commercial buffers, such as Starting Block (Thermo Fisher), were also tested but produced no improvement in the results.

The selected blocking, 1\% BSA ON, was also tested on the Dynabeads MB, but it produced no improvement compared to the 1-h blocking (Fig S-3b). Therefore, this blocking strategy was discarded for Dynabeads.

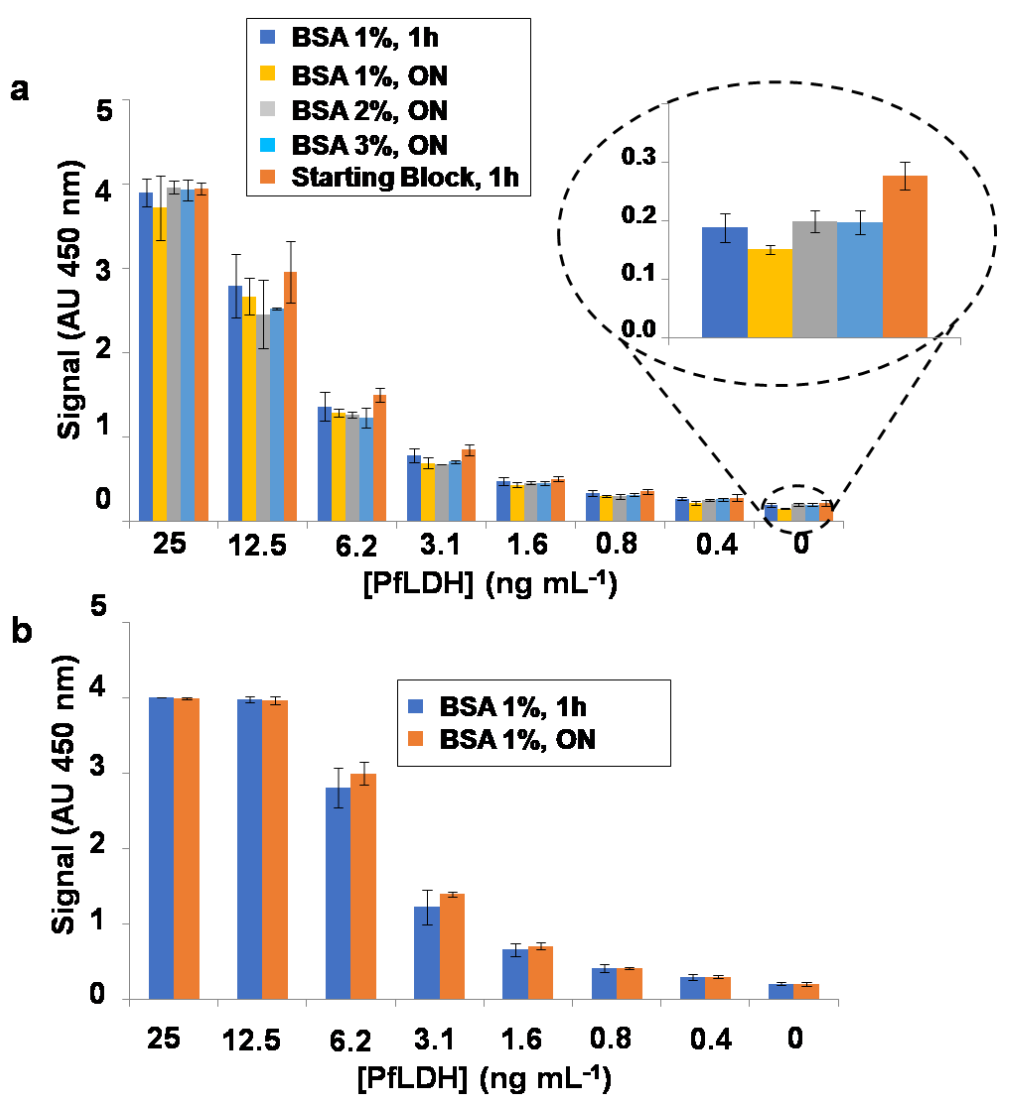


Figure S5. Performance of the 2-step magneto-immunoassay in lysed whole blood.

The colorimetric 2-step magneto-immunoassay was used to detect increasing concentrations of PfLDH spiked in different sample matrices. For this, whole blood was mixed 1:2 with lysis buffer, was lysed for $5 \mathrm{~min}$, was diluted with PBS-BSA1\% (1:5, 1:12.5, 1:25 and 1:50) to achieve final blood dilutions 1:10, 1:25, 1:50 and 1:100, and was spiked with known concentrations of PfLDH. As it can be seen in Figure S-5a, the background noise increased proportionally to sample complexity, affecting also the signals registered for all the concentrations of spiked PfLDH analysed. If the signals obtained for spiked PfLDH were corrected by subtracting the signals registered for the corresponding blanks (diluted lysed blood without spiked PfLDH), all blood dilutions produced comparable signals (Figure S-5c) and assay sensitivity (Figure S-5c, insert). Nevertheless, the high background noise contributed to decrease the $S / N$, especially for blood diluted 1:10 - 1:25 (Figure S-5b). On the other hand, PfLDH recovery ranged 70-119\%, 80-120\%, 88-114 \%, and 92-124\% for lysed whole blood diluted 1:10, 1:25, 1:50 and 1:100, respectively.

Figure S-5d displays the calibration plots obtained with the chemiluminescent 2-step magnetoimmunoassay when detecting increasing concentrations of PfLDH spiked alternatively in PBSBSA1\% and in lysed whole blood diluted 1:50. Although the background signals registered in lysed whole blood were higher than those obtained in PBS-BSA1\%, this did not affect the signals registered for spiked PfLDH and assay performance was not strongly affected by the lysed sample components. Therefore, this blood dilution was selected for the analysis of patient samples due to the low impact of the matrix in the $\mathrm{S} / \mathrm{N}$ and in protein recovery.
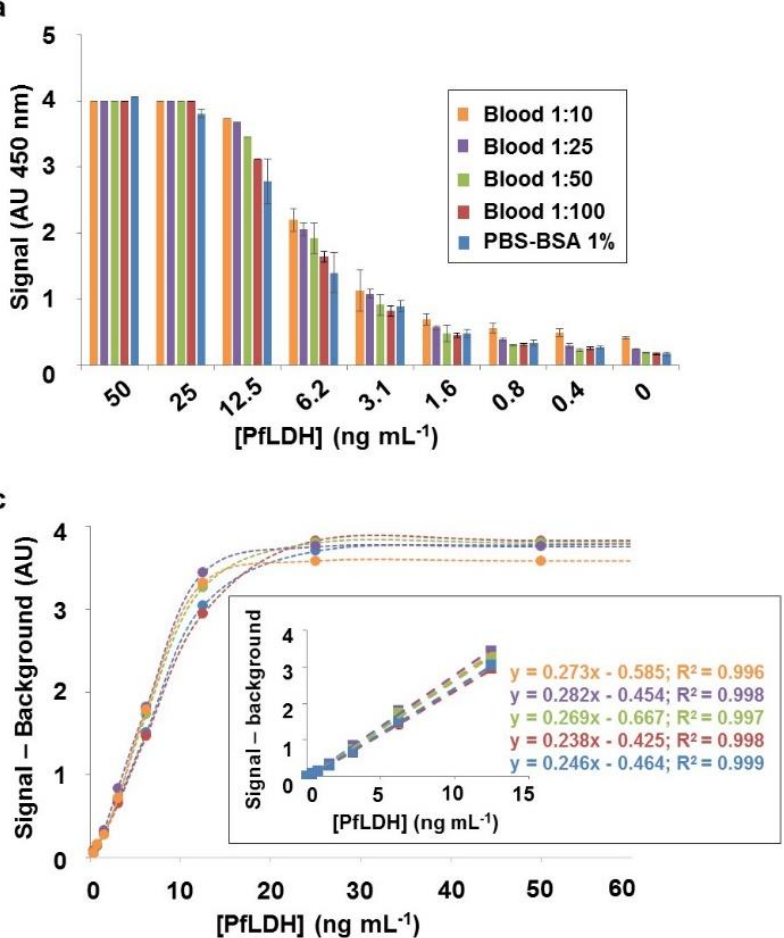

b

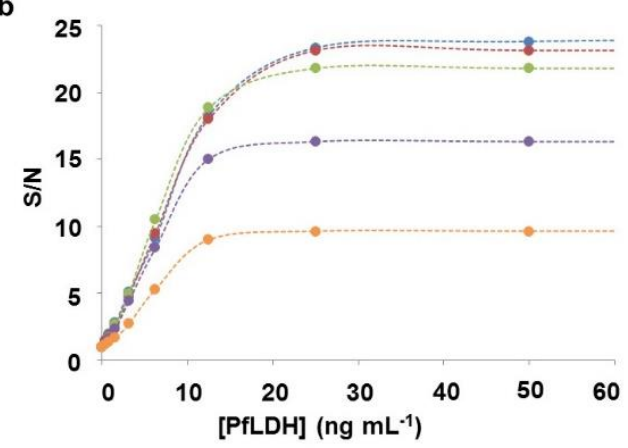

d

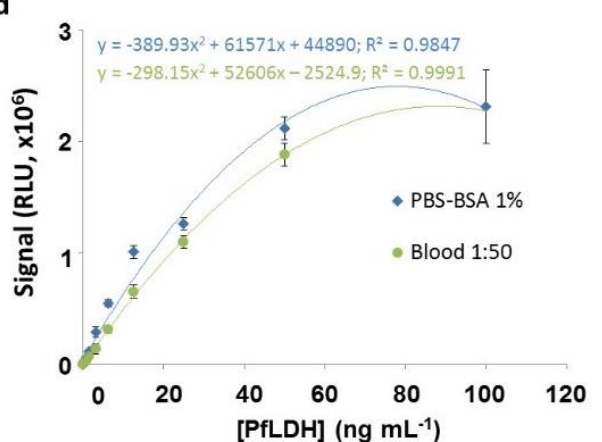




\section{References}

(1) Markwalter, C. F.; Davis, K. M.; Wright, D. W. Immunomagnetic capture and colorimetric detection of malarial biomarker Plasmodium falciparum lactate dehydrogenase. Anal. Biochem. 2016, 493, 30-34.

(2) Markwalter, C. F.; Ricks, K. M.; Bitting, A. L.; Mudenda, L.; Wright, D. W. Simultaneous capture and sequential detection of two malarial biomarkers on magnetic microparticles. Talanta 2016, 161, 443-449.

(3) De Souza Castilho, M.; Laube, T.; Yamanaka, H.; Alegret, S.; Pividori, M. I. Magneto immunoassays for plasmodium falciparum histidine-rich protein 2 related to malaria based on magnetic nanoparticles. Anal. Chem. 2011, 83 (14), 5570-5577.

(4) Kim, C.; Searson, P. C. Detection of Plasmodium Lactate Dehydrogenase Antigen in Buffer Using Aptamer-Modified Magnetic Microparticles for Capture, Oligonucleotide-Modified Quantum Dots for Detection, and Oligonucleotide-Modified Gold Nanoparticles for Signal Amplification. Bioconjug. Chem. 2017, 28 (9), 2230-2234.

(5) Ruiz-Vega, G.; Arias-Alpízar, K.; de la Serna, E.; Borgheti-Cardoso, L. N.; Sulleiro, E.; Molina, I.; Fernàndez-Busquets, X.; Sánchez-Montalvá, A.; del Campo, F. J.; Baldrich, E. Electrochemical POC device for fast malaria quantitative diagnosis in whole blood by using magnetic beads, Poly-HRP and microfluidic paper electrodes. Biosens. Bioelectron. 2020, 150, 111925.

(6) Sánchez-Tirado, E.; González-Cortés, A.; Yáñez-Sedeño, P.; Pingarrón, J. M. Carbon nanotubes functionalized by click chemistry as scaffolds for the preparation of electrochemical immunosensors. Application to the determination of TGF-beta 1 cytokine. Analyst 2016, 141 (20), 5730-5737.

(7) Sánchez-Tirado, E.; Salvo, C.; González-Cortés, A.; Yáñez-Sedeño, P.; Langa, F.; Pingarrón, J. $M$. Electrochemical immunosensor for simultaneous determination of interleukin-1 beta and tumor necrosis factor alpha in serum and saliva using dual screen printed electrodes modified with functionalized double-walled carbon nanotubes. Anal. Chim. Acta 2017, 959, $66-73$.

(8) Carvajal, S.; Fera, S. N.; Jones, A. L.; Baldo, T. A.; Mosa, I. M.; Rusling, J. F.; Krause, C. E. Disposable inkjet-printed electrochemical platform for detection of clinically relevant HER2 breast cancer biomarker. Biosens. Bioelectron. 2018, 104, 158-162.

(9) Martínez-Periñán, E.; Sánchez-Tirado, E.; González-Cortés, A.; Barderas, R.; SánchezPuelles, J. M.; Martínez-Santamaría, L.; Campuzano, S.; Yáñez-Sedeño, P.; Pingarrón, J. M. Amperometric determination of endoglin in human serum using disposable immunosensors constructed with poly(pyrrolepropionic) acid-modified electrodes. Electrochim. Acta 2018, 292, 887-894.

(10) de la Serna, E.; Martínez-García, E.; García-Berrocoso, T.; Penalba, A.; Gil-Moreno, A.; Colas, E.; Montaner, J.; Baldrich, E. Using polyHRP to produce simplified immunoassays and electrochemical immunosensors. Application to MMP-9 detection in plasma and uterine aspirates. Sensors Actuators, B Chem. 2018, 269, 377-384.

(11) Sánchez-Tirado, E.; Martínez-García, G.; González-Cortés, A.; Yáñez-Sedeño, P.; Pingarrón, J. M. Electrochemical immunosensor for sensitive determination of transforming growth factor (TGF) - $\beta 1$ in urine. Biosens. Bioelectron. 2017, 88, 9-14.

(12) Arévalo, B.; ben Hassine, A.; Valverde, A.; Serafín, V.; Montero-Calle, A.; Raouafi, N.; Camps, J.; Arenas, M.; Barderas, R.; Yáñez-Sedeño, P.; Campuzano, S.; Pingarrón, J. M. Electrochemical immunoplatform to assist in the diagnosis and classification of breast 
cancer through the determination of matrix-metalloproteinase-9. Talanta 2021, 225, 122054.

(13) Ruiz-Vega, G.; García-Robaina, A.; Ben Ismail, M.; Pasamar, H.; García-Berrocoso, T.; Montaner, J.; Zourob, M.; Othmane, A.; del Campo, F. J.; Baldrich, E. Detection of plasma MMP-9 within minutes. Unveiling some of the clues to develop fast and simple electrochemical magneto-immunosensors. Biosens. Bioelectron. 2018, 115, 45-52.

(14) Tran, T. V.; Nguyen, B. V.; Nguyen, T. T. P.; Tran, T. T.; Pham, K. G.; Le, Q. B.; Do, B. N.; Pham, H. N.; Nguyen, C. V.; Dinh, D. P. H.; Ha, V. T.; Doan, T. H. T.; Le, H. Q. Development of a highly sensitive magneto-enzyme lateral flow immunoassay for dengue NS1 detection. PeerJ 2019, 7, e7779.

(15) de la Serna, E.; Arias-Alpízar, K.; Borgheti-Cardoso, L. N.; Sanchez-Cano, A.; Sulleiro, E.; Zarzuela, F.; Bosch-Nicolau, P.; Salvador, F.; Molina, I.; Ramírez, M.; Fernàndez-Busquets, X.; Sánchez-Montalvá, A.; Baldrich, E. Detection of Plasmodium falciparum malaria in $1 \mathrm{~h}$ using a simplified enzyme-linked immunosorbent assay. Anal. Chim. Acta 2021, 1152, 338254.

(16) Ben Ismail, M.; de la Serna, E.; Ruiz-Vega, G.; García-Berrocoso, T.; Montaner, J.; Zourob, M.; Othmane, A.; Baldrich, E. Using magnetic beads and signal amplifiers to produce short and simple immunoassays: Application to MMP-9 detection in plasma samples. Anal. Chim. Acta 2018, 999, 144-154. 